\title{
Efectos de la criopreservación sobre las subpoblaciones espermáticas en caprinos
}

\author{
EFFECTS OF CRYOPRESERVATION ON SPERM SUBPOPULATIONS IN GOATS \\ Leonardo Hernández Corredor ${ }^{1,3,4,6}$, Omar Camargo Rodríguez ${ }^{2}$, Albeiro Silva \\ Torres ${ }^{4}$, Juan David Montoya Páez ${ }^{5}$, Armando Quintero Moreno ${ }^{1}$
}

\section{Resumen}

El objetivo de este estudio fue evaluar el efecto de la criopreservación en la presencia de subpoblaciones de espermatozoides, según la motilidad, en eyaculados de macho cabrío utilizando el sistema de Análisis de Semen Asistido por Computador (CASA). Los parámetros de motilidad se analizaron con análisis de componentes principales (PCA) donde evidenciaron la mayor varianza, reduciendo así el número de variables. En la evaluación de 23738 espermatozoides en fresco, la subpoblación (Sp) 1 consistió en espermatozoides progresivos y mediana progresividad (18.34\%), la $\mathrm{Sp} 2$ en espermatozoides de alta velocidad y progresivos (20.53\%), la Sp 3 en espermatozoides de alta actividad, pero no progresivos $(46.79 \%)$ y la $\mathrm{Sp} 4$ en espermatozoides poco activos y no progresivos (14.32\%), habiendo diferencias significativas en la distribución de las cuatro Sp $(\mathrm{p}<0.001)$. La estructura de la $\mathrm{Sp}$ de espermatozoides no se mantuvo después del almacenamiento en frío. Al evaluar los mismos parámetros en muestras descongeladas en 36450 espermatozoides móviles, la Sp 1 consistió en espermatozoides progresivos bajos y lineales lentos (38.43\%), la Sp 2 en espermatozoides poco activos y progresivos lentos $(7.3 \%)$, la $\mathrm{Sp} 3$ en células espermáticas con alta actividad y excelente progresividad (11.65\%) y la Sp 4 en espermatozoides activos, pero no progresivos $(42.61 \%)$, habiendo diferencias significativas en la distribución de las cuatro $\mathrm{Sp}(\mathrm{p}<0.001)$. La criopreservación modificó significativamente tanto los parámetros específicos como la distribución de los espermatozoides dentro de las subpoblaciones.

Palabras clave: espermatozoides; cabras; motilidad; CASA; poblaciones

\footnotetext{
${ }^{1}$ Unidad de Investigación en Producción Animal (UNIPA), Facultad de Ciencias Veterinarias (FCV), Universidad del Zulia (LUZ), Maracaibo, Venezuela

${ }^{2}$ Universidad Nacional de Colombia, Medellín, Colombia

${ }^{3}$ Universidad Francisco de Paula Santander-SENNOVA, SENA-Norte de Santander, Cúcuta, Colombia

${ }^{4}$ Universidad de Pamplona, Pamplona, Colombia

${ }^{5}$ Politécnico Colombiano Jaime Isaza Cadavid, Medellín, Colombia

${ }^{6}$ E-mail: lehernandez@fa.luz.edu.ve; lhernandezc@sena.edu.co
} 
The aim of this study was to evaluate the effect of cryopreservation in the presence of sperm subpopulations, according to motility, in sperm ejaculates using the Computer Assisted Semen Analysis (CASA) system. The motility parameters were analysed with principal component analysis (PCA) where they showed the highest variance, thus reducing the number of variables. In the evaluation of 23738 fresh spermatozoa, the subpopulation ( $\mathrm{Sp}$ ) 1 consisted of progressive spermatozoa and median progressive motility (18.34\%), Sp 2 in high velocity and progressive spermatozoa (20.53\%), Sp 3 in high-activity spermatozoa, but not progressive (46.79\%) and Sp 4 in little activity and non-progressive spermatozoa (14.32\%), showing significant differences in the distribution of the four $\mathrm{Sp}(\mathrm{p}<0.001)$. The structure of the spermatozoa $\mathrm{Sp}$ was not maintained after freezing. When evaluating the same parameters in thawed samples in 36450 motile sperm, the $\mathrm{Sp} 1$ consisted of slow and linear slow progressive spermatozoa (38.43\%), the Sp 2 in slowly active and slow spermatozoa (7.3\%), the Sp 3 in spermatozoa with high activity and excellent progressive motility (11.65\%) and $\mathrm{Sp} 4$ in active spermatozoa, but not progressive (42.61\%), showing significant differences in the distribution of the four Sp $(\mathrm{p}<0.001)$. Cryopreservation significantly modified both the specific parameters and the distribution of the spermatozoa within the subpopulations.

Key words: spermatozoa; goat; motility; CASA; population; cryopreservation

\section{INTRODUCCIÓN}

La inseminación artificial (IA) es una biotecnología reproductiva que para ser efectiva depende de la calidad seminal. En el caso de los caprinos, su procesamiento (dilución, enfriamiento y conservación) presenta algunas dificultades que determinan una menor eficiencia en comparación con otras especies (bovina, ovina). Esto es debido a la presencia de ciertas sustancias que forman parte del plasma seminal, las cuales reaccionan con algunos componentes de uso común en los diluyentes de semen (Dorado, 2003; Hidalgo et al., 2006, Dorado et al., 2010; Salomón, 2011).

Desde la época en que se demostró la existencia de subpoblaciones espermáticas en los eyaculados frescos y conservados de mamíferos (Holt, 1996; Abaigar et al., 1999; Thurston et al., 2001; Quintero-Moreno, 2003; Dorado, 2003; Martínez-Pastor et al., 2005), se ha intentado generar explicaciones que puedan predecir sus patrones de movimien- to, incluyendo la raza, temperatura ambiental, época del año y estado nutricional, entre otros.

Desde los primeros años del CASA (Computer-assisted sperm analysis), los datos se trabajaban con análisis multivariados con un alto grado de precisión (QuinteroMoreno, 2003), aunque la subjetividad todavía estaba presente en la definición de las subpoblaciones al utilizar métodos jerárquicos. Martínez-Pastor et al. (2005) introducen un método no jerárquico (FASTCLUS) donde tiene en cuenta la pseudo $t^{2}$, la pseudo F y el criterio cúbico clusterizado (CCC) para determinar el número final de cluster, logrando una mejor clasificación de subpoblaciones espermáticas, siendo objetivo a los datos entregados por el software.

El objetivo de este estudio fue comprender las subpoblaciones espermáticas en el contenido seminal de machos cabríos y el efecto de la criopreservación sobre la motilidad. 


\section{Materiales y Métodos}

\section{Animales y Colecta de Semen}

Se emplearon siete machos cabríos adultos (4 alpinos y 3 Saanen), entre 3 y 5 años de edad, de reconocida capacidad reproductiva. Los animales fueron sometidos a un ritmo de colectas de dos veces por semana.

Se recolectaron 56 eyaculados (ocho por macho) durante un periodo de dos meses, empleando una vagina artificial (Walmur $^{\circledR}$, Porto Alegre, Brasil). Los eyaculados se llevaron al Laboratorio de Biotecnología Animal del Politécnico Colombiano Jaime Isaza Cadavid, sede Bello (Antioquia, Colombia), donde fueron mantenidos en baño maría a $37^{\circ} \mathrm{C}$ hasta su valoración.

\section{Evaluación en Fresco y Criopreser- vación}

Se evaluó la motilidad espermática por el sistema CASA (Sperm Class Analyzer, $\mathrm{SCA}^{\circledR}$, Microptic, Barcelona, España). El análisis se realizó sobre alícuotas de $5 \mu 1$ de semen fresco a $37^{\circ} \mathrm{C}$. Las alícuotas fueron colocadas en láminas Leja ${ }^{\circledR}$ para la medición de la motilidad por el sistema CASA. Luego, se capturaron las imágenes de los espermatozoides en movimiento con un microscopio de contraste de fase (Eclipse E200, Nikon, Japón) a 200X. Los parámetros evaluados fueron Velocidad curvilínea (VCL, $\mu \mathrm{m} / \mathrm{s}$ ), Velocidad rectilínea (VSL, $\mu \mathrm{m} / \mathrm{s}$ ), Velocidad media (VAP, $\mu \mathrm{m} / \mathrm{s}$ ), Índice de linealidad (LIN, \%), Índice de rectitud (STR, $\%$ ), Índice de oscilación (WOB, \%) y Frecuencia de batida de la cabeza (BCF, Hz). Estos parámetros cinéticos han sido previamente descritos por Mortimer (1997) y Quintero-Moreno (2003).

Las muestras seminales fueron diluidas en dos alicuotas en una proporción 1:1 de Biladyl ${ }^{\circledR}$ y centrifugadas a $800 g$ durante 3 min
(Dynac III ${ }^{\circledR}$, Becton Dickinson, EEUU). A continuación, se retiró el sobrenadante y el pellet de espermatozoides fue re-diluido con los diluyentes comerciales: AndroMed ${ }^{\circledR}$ (Minitüb GmbH, Tiefenbach, Alemania) y Triladyl ${ }^{\circledR}$ (Minitüb GmbH, Tiefenbach, Alemania). La concentración final aproximada fue de $100 \times 10^{6}$ espermatozoides $/ \mathrm{ml}$. Las muestras fueron empacadas y selladas (MRS1 Dual V2, IMV Technologies, Francia) en pajuelas de $0.50 \mathrm{ml}$ y refrigeradas a $4{ }^{\circ} \mathrm{C}$ durante $2 \mathrm{~h}$. Finalmente, las pajuelas de cada tratamiento fueron congeladas en vapores de nitrógeno líquido, colocándolas a 4 $\mathrm{cm}$ de la superficie del nitrógeno durante 15 min para seguidamente ser sumergidas en nitrógeno líquido $\left(-196^{\circ} \mathrm{C}\right)$.

\section{Análisis Objetivo del Movimiento Espermático}

Para el análisis del semen congelado, se evaluaron cinco pajuelas por colecta, según la metodología descrita por Dorado et al. (2007) para el macho cabrío. Las pajuelas fueron elegidas al azar y descongeladas en baño maría a $37^{\circ} \mathrm{C}$ durante $30 \mathrm{~s}$. Las muestras descongeladas fueron diluidas con PBS hasta una concentración de 1 x $10^{6}$ espermatozoides $/ \mathrm{ml}$. Se hicieron alícuotas de $5 \mu 1$ de semen diluido que se depositaron en cámaras de un solo uso Leja ${ }^{\circledR}(20 \mu \mathrm{m}-4$ cámaras, SC-20-01-04-B, Países Bajos). En cada análisis, un total de cinco campos microscópicos fueron aleatoriamente seleccionados, con la misma metodología empleada para el semen fresco.

\section{Análisis Estadístico}

Se utilizó estadística descriptiva (medias, desviaciones estándar). Se aplicó una prueba de normalidad y análisis de varianza, bajo un diseño experimental factorial donde se evaluaron los efectos de los diluyentes y la raza sobre las variables de motilidad seminal. Del mismo modo y con el propósito de identificar la presencia de subpoblaciones espermáticas en el eyaculado, los valores de la motilidad fueron sometidas a un análisis 
multivariado de agrupamiento no jerárquico, el cual agrupó a los espermatozoides según sus características motiles comunes, como se ha descrito anteriormente (Quintero-Moreno et al., 2003).

Bajo la metodología de Martínez-Pastor et al. (2004), se utilizó un análisis de componentes principales (PRINCOMP) como primer paso, para seleccionar las variables del eigenvalue (varianzas extraídas de los componentes principales, [Criterio Kaiser]); en un segundo paso se ejecutó un clúster no jerárquico (FASTCLUS) y se hizo un análisis de conglomerados utilizando una distancia euclidiana para determinar los centroides, reduciendo los datos. Luego se determinó un procedimiento jerárquico, seleccionando un máximo de 15 grupos, ya que este número es adecuado para hacer el procedimiento jerárquico posterior (se pueden presentar errores al final del agrupamiento si se usan grupos iniciales muy pequeños). En el caso de detectar valores atípicos (valores con un solo dato) se suprimen los valores extremos y se ejecuta nuevamente el programa. En la tercera parte se hizo un dendograma usando el método de ligamiento medio (UPGMA) para unir los grupos. Para determinar este grupo final se tuvo en cuenta los criterios del cluster: la pseudo- $\mathrm{t}^{2}$, el pseudo F y el criterio de agrupamiento cúbico (CCC). Se buscan los valores altos de la CCC y pseudo F en combinación de un valor con tendencia a bajar y un pseudo- $\mathrm{t}^{2} \mathrm{y}$ un valor más grande pseudo$\mathrm{t}^{2}$ para la próxima fusión clúster.

Después del análisis de agrupamiento, se compararon los tratamientos dentro de las subpoblaciones y subpoblaciones dentro de los tratamientos (que incluyen las agrupaciones y toda la población de espermatozoides por motilidad dentro del término subpoblación). Se utilizó el procedimiento GLM (modelos lineales generales) para llevar a cabo una ANOVA de la viabilidad y el estado acrosomal antes y después de la criopreservación. Los datos con porcentajes fueron sometidos a transformación arco seno y medidas absolutas para registrar la transformación.
Para cualificar la relación existente entre los diluyentes y la frecuencia de distribución de los espermatozoides de cada subpoblación se analizaron los datos mediante la prueba de Chi-cuadrado. Los datos fueron analizados mediante el Statistical Analysis System v. 12.0 para Windows (SAS Inst. Inc.; Carry, NC, EEUU), con el Modelo Lineal General del Análisis de la Varianza (Proc GLM). Cuando se encontraron diferencias entre las medias se cuantificó el efecto mediante el procedimiento LSMEANS, FASTCLUS.

\section{Resultados}

\section{Motilidad del Semen}

Se estudiaron ocho variables (VCL, VSL, VAP, LIN, STR, WOB, ALH, BCF) en 23738 espermatozoides en el semen fresco y 36450 espermatozoides en el semen descongelado por medio del sistema CASA. En el Cuadro 1 se observa los parámetros de motilidad para semen fresco obtenidos por el sistema CASA. Se observaron diferencias en las velocidades (VCL, VSL) y en los parámetros de calidad de movimientos (LIN y STR), tanto en las muestras de semen fresco como en las muestras de semen descongelado $(\mathrm{p}<0.05)$, siendo la raza Alpina la que mostró un valor más alto.

\section{Subpoblaciones Espermáticas}

Se aplicó a los espermatozoides un análisis de componentes principales (ACP) para reducir las variables cinéticas primarias a tres componentes (Prin1, Prin2, Prin3). En el semen fresco explicaron el $86.75 \%$ de las diferencias. La Prin1 se relaciona con el movimiento espermático y explica el $42.07 \%$ de la varianza, la Prin2 se relaciona con la linealidad y explica el $31.69 \%$ de la varianza, y la Prin 3 se relaciona con la actividad flagelar y explica el $12.98 \%$ de la varianza. En el semen descongelado explicaron el $88.97 \%$, donde la Prin1 explica el $43.05 \%$ de la 
Cuadro 1. Descriptores ${ }^{1}$ de motilidad obtenidos de 56 eyaculados en siete reproductores caprinos (23 738 y 36450 espermatozoides para semen fresco y congelado, respectivamente)

\begin{tabular}{|c|c|c|c|c|c|c|c|c|c|}
\hline $\begin{array}{l}\text { Semen / } \\
\text { Raza }\end{array}$ & $\%$ & $\begin{array}{c}\text { VCL } \\
(\mu \mathrm{m} / \mathrm{s})\end{array}$ & $\begin{array}{c}\mathrm{VSL} \\
(\mu \mathrm{m} / \mathrm{s})\end{array}$ & $\begin{array}{c}\text { VAP } \\
(\mu \mathrm{m} / \mathrm{s})\end{array}$ & $\begin{array}{l}\text { LIN } \\
(\%)\end{array}$ & $\begin{array}{l}\text { STR } \\
(\%)\end{array}$ & $\begin{array}{l}\text { WOB } \\
(\%)\end{array}$ & $\begin{array}{c}\text { ALH } \\
(\mu \mathrm{m} / \mathrm{s})\end{array}$ & $\begin{array}{l}\mathrm{BCF} \\
(\mathrm{Hz})\end{array}$ \\
\hline \multicolumn{10}{|l|}{ Fresco } \\
\hline Alpino & 66.1 & $\begin{array}{l}123.65 \\
\pm 0.42^{\mathrm{a}}\end{array}$ & $\begin{array}{l}46.95 \\
\pm 0.25^{\mathrm{a}}\end{array}$ & $\begin{array}{c}83.52 \\
\pm 0.37^{\mathrm{a}}\end{array}$ & $\begin{array}{c}35.57 \\
\pm 0.16^{\mathrm{a}}\end{array}$ & $\begin{array}{c}53.37 \\
\pm 0.19^{\mathrm{a}}\end{array}$ & $\begin{array}{c}65.15 \\
\pm 0.14^{\mathrm{b}}\end{array}$ & $\begin{array}{c}4.66 \\
\pm 0.01^{\mathrm{a}}\end{array}$ & $\begin{array}{c}5.45 \\
\pm 0.02^{\mathrm{b}}\end{array}$ \\
\hline Saanen & 33.9 & $\begin{array}{l}122.91 \\
\pm 0.53^{b}\end{array}$ & $\begin{array}{c}43.07 \\
\pm 0.35^{\mathrm{b}}\end{array}$ & $\begin{array}{c}87.98 \\
\pm 0.53^{\mathrm{a}}\end{array}$ & $\begin{array}{c}35.08 \\
\pm 0.23^{\mathrm{b}}\end{array}$ & $\begin{array}{c}50.50 \\
\pm 0.27^{\mathrm{b}}\end{array}$ & $\begin{array}{c}68.64 \\
\pm 0.18^{\mathrm{a}}\end{array}$ & $\begin{array}{c}4.37 \\
\pm 0.02^{\mathrm{b}}\end{array}$ & $\begin{array}{c}5.82 \\
\pm 0.03^{\mathrm{a}}\end{array}$ \\
\hline Total & 100 & $\begin{array}{l}123.40 \\
\pm 0.33\end{array}$ & $\begin{array}{l}43.65 \\
\pm 0.21\end{array}$ & $\begin{array}{l}85.03 \\
\pm 0.30\end{array}$ & $\begin{array}{l}35.41 \\
\pm 0.13\end{array}$ & $\begin{array}{l}52.40 \\
\pm 0.16\end{array}$ & $\begin{array}{l}66.37 \\
\pm 0.10\end{array}$ & $\begin{array}{c}4.56 \\
\pm 0.01\end{array}$ & $\begin{array}{c}5.58 \\
\pm 0.02\end{array}$ \\
\hline \multicolumn{10}{|c|}{ Descongelado } \\
\hline Alpino & 68.8 & $\begin{array}{l}126.33 \\
\pm 5.31^{\mathrm{a}}\end{array}$ & $\begin{array}{c}46.33 \\
\pm 2.89^{b}\end{array}$ & $\begin{array}{c}88.93 \\
\pm 5.28^{\mathrm{b}}\end{array}$ & $\begin{array}{c}36.53 \\
\pm 1.57^{\mathrm{b}}\end{array}$ & $\begin{array}{c}52.77 \\
\pm 1.72^{\mathrm{a}}\end{array}$ & $\begin{array}{c}68.95 \\
\pm 1.90^{\mathrm{b}}\end{array}$ & $\begin{array}{c}3.91 \\
\pm 0.13^{\mathrm{b}}\end{array}$ & $\begin{array}{c}6.43 \\
\pm 0.24^{\mathrm{a}}\end{array}$ \\
\hline Saanen & 31.2 & $\begin{array}{l}125.11 \\
\pm 7.82^{\mathrm{b}}\end{array}$ & $\begin{array}{c}51.27 \\
\pm 7.71^{\mathrm{a}}\end{array}$ & $\begin{array}{c}96.93 \\
\pm 9.58^{\mathrm{a}}\end{array}$ & $\begin{array}{c}40.08 \\
\pm 4.32^{\mathrm{a}}\end{array}$ & $\begin{array}{c}52.49 \\
\pm 3.86^{\mathrm{a}}\end{array}$ & $\begin{array}{c}75.92 \\
\pm 3.83^{\mathrm{a}}\end{array}$ & $\begin{array}{c}3.22 \\
\pm 0.27^{\mathrm{a}}\end{array}$ & $\begin{array}{c}6.53 \\
\pm 0.50^{\mathrm{a}}\end{array}$ \\
\hline Total & 100 & $\begin{array}{l}125.93 \\
\pm 4.32\end{array}$ & $\begin{array}{l}47.98 \\
\pm 3.16\end{array}$ & $\begin{array}{l}91.60 \\
\pm 4.71\end{array}$ & $\begin{array}{l}37.72 \\
\pm 1.76\end{array}$ & $\begin{array}{l}52.64 \\
\pm 1.69\end{array}$ & $\begin{array}{l}71.27 \\
\pm 1.85\end{array}$ & $\begin{array}{c}3.68 \\
\pm 0.13\end{array}$ & $\begin{array}{c}6.46 \\
\pm 0.22\end{array}$ \\
\hline
\end{tabular}

a,b Superíndices dentro de columnas para semen fresco y semen descongelado indican diferencia significativa entre razas $(p<0.05)$

${ }^{1} \mathrm{VCL}=$ velocidad curvilínea; $\mathrm{VSL}=$ velocidad rectilínea; $\mathrm{VAP}=$ velocidad de la trayectoria media; LIN = linealidad; STR = rectitud; $W O B=$ índice de oscilación; $A L H=$ amplitud media del desplazamiento lateral de la cabeza; $\mathrm{BCF}=$ frecuencia de batido (valores: media \pm error estándar de la media)

varianza, la Prin 2 el $35.95 \%$ de la varianza y la Prin3 el 9.97\% de la varianza (Cuadro 2).

En las subpoblaciones (sp) de espermatozoides frescos:

- Sp1. El 18.34\% de la población son espermatozoides progresivos con el mayor porcentaje de motilidad progresiva (valores muy elevados de VSL, VAP, LIN y STR junto a valores medios de ALH), probablemente con el patrón de descriptores más adecuado para formar parte de la población de fertilización.

- Sp2. El 20.53\% de la población son espermatozoides muy activos, pero no progresivos (valores altos de VCL, ALH, LIN y STR). Los bajos valores de LIN indican que estas células muestran movimientos circulares o irregulares. Asimismo, los valores elevados de VCL y ALH indican que esta subpoblación podría ser considerada como espermatozoides con movimiento muy similares a la hiperactivación.

- Sp3. El 46.79\% de la población incluyó espermatozoides lentos, pero con alta actividad espermática (valores reducidos de VCL y medios de VAP), con trayectorias progresivas (valores buenos de LIN, STR y WOB). Además, son espermatozoides que recorren distancias medias (valores medios de VSL).

- Sp4. El $14.32 \%$ de la población son espermatozoides cuyo movimiento fue menos vigoroso, (valores más bajos de VCL, VSL, VAP y ALH). Presentaron escasa progresividad (valores bajos de VAP, LIN y STR), recorrieron distancias 
Cuadro 2. Valores de motilidad de subpoblaciones espermáticas obtenidos de 56 eyaculados de siete reproductores caprinos en semen fresco y descongelado

\begin{tabular}{lcccc}
\hline & \multicolumn{4}{c}{ Subpoblaciones } \\
\cline { 2 - 5 } Descriptores & Sp1 & Sp2 & Sp3 & Sp4 \\
\cline { 2 - 5 } Semen fresco & & & \\
VCL $(\mu \mathrm{m} / \mathrm{s})$ & $173.21 \pm 0.36^{\mathrm{a}}$ & $171.76 \pm 0.41^{\mathrm{a}}$ & $97.87 \pm 0.35^{\mathrm{b}}$ & $73.65 \pm 0.48^{\mathrm{c}}$ \\
VSL $(\mu \mathrm{m} / \mathrm{s})$ & $83.99 \pm 0.39^{\mathrm{a}}$ & $31.04 \pm 0.26^{\mathrm{c}}$ & $43.56 \pm 0.26^{\mathrm{b}}$ & $10.38 \pm 0.11^{\mathrm{d}}$ \\
VAP $(\mu \mathrm{m} / \mathrm{s})$ & $134.90 \pm 0.47^{\mathrm{a}}$ & $11.69 \pm 0.61^{\mathrm{d}}$ & $68.89 \pm 0.32^{\mathrm{b}}$ & $35.68 \pm 0.30^{\mathrm{c}}$ \\
LIN $(\%)$ & $48.27 \pm 0.001^{\mathrm{a}}$ & $17.46 \pm 0.001^{\mathrm{b}}$ & $44.80 \pm 0.001^{\mathrm{a}}$ & $13.95 \pm 0.001^{\mathrm{b}}$ \\
STR $(\%)$ & $63.58 \pm 0.002^{\mathrm{a}}$ & $29.39 \pm 0.002^{\mathrm{b}}$ & $64.75 \pm 0.20^{\mathrm{a}}$ & $30.73 \pm 0.002^{\mathrm{b}}$ \\
WOB $(\%)$ & $77.34 \pm 0.001^{\mathrm{a}}$ & $63.41 \pm 0.002^{\mathrm{b}}$ & $68.98 \pm 0.001^{\mathrm{b}}$ & $47.76 \pm 0.002^{\mathrm{c}}$ \\
ALH $(\mu \mathrm{m} / \mathrm{s})$ & $5.32 \pm 0.01^{\mathrm{b}}$ & $6.48 \pm 0.01^{\mathrm{a}}$ & $3.70 \pm 0.01^{\mathrm{c}}$ & $3.64 \pm 0.02^{\mathrm{c}}$ \\
BCF $(\mathrm{Hz})$ & $6.14 \pm 0.04^{\mathrm{a}}$ & $4.92 \pm 0.04^{\mathrm{c}}$ & $5.86 \pm 0.02^{\mathrm{b}}$ & $4.87 \pm 0.04^{\mathrm{c}}$ \\
\hline Total de la muestra N & 4,354 & 4,876 & $11,108^{\mathrm{b}}$ & 3,400 \\
$(\%)$ & $(18.34)$ & $(20.53)$ & $(46.79)$ & $(14.32)$ \\
\hline Semen descongelado & & & & \\
VCL $(\mu \mathrm{m} / \mathrm{s})$ & $107.64 \pm 0.34^{\mathrm{a}}$ & $98.12 \pm 0.72^{\mathrm{b}}$ & $62.19 \pm 0.54^{\mathrm{c}}$ & $35.01 \pm 0.11^{\mathrm{d}}$ \\
VSL $(\mu \mathrm{m} / \mathrm{s})$ & $62.03 \pm 0.29^{\mathrm{a}}$ & $18.72 \pm 0.28^{\mathrm{c}}$ & $52.73 \pm 0.51^{\mathrm{b}}$ & $10.57 \pm 0.06^{\mathrm{d}}$ \\
VAP $(\mu \mathrm{m} / \mathrm{s})$ & $85.57 \pm 0.33^{\mathrm{a}}$ & $59.33 \pm 0.72^{\mathrm{b}}$ & $56.95 \pm 0.53^{\mathrm{c}}$ & $20.13 \pm 0.08^{\mathrm{d}}$ \\
LIN $(\%)$ & $56.74 \pm 0.16^{\mathrm{b}}$ & $17.94 \pm 0.19^{\mathrm{d}}$ & $82.56 \pm 0.16^{\mathrm{a}}$ & $29.89 \pm 0.16^{\mathrm{c}}$ \\
STR $(\%)$ & $72.50 \pm 0.17^{\mathrm{b}}$ & $33.16 \pm 0.39^{\mathrm{d}}$ & $91.27 \pm 0.11^{\mathrm{a}}$ & $50.58 \pm 0.21^{\mathrm{c}}$ \\
WOB $(\%)$ & $78.16 \pm 0.11^{\mathrm{b}}$ & $57.58 \pm 0.35^{\mathrm{c}}$ & $90.36 \pm 0.12^{\mathrm{a}}$ & $57.09 \pm 0.14^{\mathrm{c}}$ \\
ALH $(\mu \mathrm{m} / \mathrm{s})$ & $3.47 \pm 0.09^{\mathrm{b}}$ & $4.21 \pm 0.02^{\mathrm{a}}$ & $1.73 \pm 0.08^{\mathrm{d}}$ & $1.92 \pm 0.05^{\mathrm{c}}$ \\
BCF $(\mathrm{Hz})$ & $7.00 \pm 0.02^{\mathrm{a}}$ & $5.38 \pm 0.04^{\mathrm{d}}$ & $6.38 \pm 0.03^{\mathrm{b}}$ & $3.73 \pm 0.01^{\mathrm{c}}$ \\
\hline Total de la muestra N & 14.008 & 2.661 & 2.912 & 10.651 \\
$(\%)$ & $(38.43)$ & $(7.30)$ & $(11.65)$ & $(42.61)$ \\
\hline
\end{tabular}

${ }^{1} \mathrm{VCL}=$ velocidad curvilínea; $\mathrm{VSL}=$ velocidad rectilínea; $\mathrm{VAP}=$ velocidad de la trayectoria media; $\mathrm{LIN}=$ linealidad; STR = rectitud; $\mathrm{WOB}$ = índice de oscilación; $\mathrm{ALH}=$ amplitud media del desplazamiento lateral de la cabeza; $\mathrm{BCF}=$ frecuencia de batido (valores: media \pm error estándar de la media)

muy cortas (VSL muy baja). La población tiene valores bajos de VCL y LIN, que indica compromiso metabólico. Los espermatozoides pierden su movilidad por completo. Además, poseerían alteraciones estructurales o cambios bioquímicos importantes que afectan al espermatozoide.

En las subpoblaciones ( $\mathrm{sp}$ ) de espermatozoides descongelados:

- Sp1. El 38.43\% de la población son espermatozoides progresivos con el mayor porcentaje de motilidad progresiva (valores muy elevados de VSL, VAP), con el patrón de motilidad adecuado para formar parte de la población de fertilización.
- Sp2. El 7.30\% de la población son espermatozoides muy activos, pero no progresivos (valores altos de VCL, ALH). Los bajos valores de LIN indican que estas células muestran movimientos circulares o irregulares; podría ser considerada como espermatozoides con movimiento de hiperactivación.

- Sp3. El 11.65\% de la población incluyó espermatozoides lentos, pero con alta actividad espermática (valores reducidos de VCL y medios de VAP), con las mejores trayectorias progresivas (valores buenos de LIN, STR y WOB). Además, son espermatozoides que recorren distancias medias (valores medios de VSL). 
- Sp4. El $42.61 \%$ de la población son espermatozoides con valores bajos de VCL y LIN. La subpoblación tiene daño metabólico. No son aptos para la fertilización.

\section{Discusión}

El estudio detallado de cada espermatozoide se inicia con los descriptores del CASA (Boyers et al., 1989; Davis y Katz, 1993; Mortimer, 2000; Quintero-Moreno, 2003), y es con base a dichos estudios que se logra entender que un eyaculado contiene una población heterogénea de espermatozoides.

Las subpoblaciones de espermatozoides se pueden identificar sobre la base de parámetros o características distintas, tales como parámetros de motilidad, morfometría y morfología, entre otros (Quintero-Moreno, 2003; Hidalgo, 2004; Dorado et al., 2010; Martínez-Pastor et al., 2011; Ramón et al., 2013; Hernández-Corredor et al., 2014; Esteso et al., 2015). Martinez-Pastor et al. (2005) encuentran que las subpoblaciones por motilidad se originan por factores físicos, mientras que Holt y Harrinson (2002) encontraron que la LIN cambiaba dependiendo de la estimulación con bicarbonato, definiendo que las subpoblaciones se originan por factores químicos.

Los resultados del estudio de motilidad medida en fresco mediante CASA indicaron diferencias significativas entre $\operatorname{razas}(\mathrm{p}<0.05)$ para las variables VCL, VSL, LIN, STR, WOB, ALH y BCF, donde la raza alpina presentó los mejores resultados. En semen descongelado se encontraron diferencias significativas entre razas $(p<0.05)$ en las variables VSL, VAP, LIN y WOB, siendo mejor los resultados para la raza Saanen. Los mejores valores de LIN y VAP en los machos Saanen podría ser la diferencia entre alta y baja fertilidad (Hernández-Corredor et al., 2013). Diferencias entre razas han sido descritas en bovinos (Hoflack et al., 2007) y en caprinos
(Chandler et al., 1988; Karagiannidis et al., 2000; Kamal et al., 2005; Zamfirescu et al., 2005), donde las variaciones genéticas podrían ser explicadas por la selección reproductiva de los caprinos. Los resultados que se obtuvieron en las subpoblaciones de motilidad representan el estado fisiológico y sus diferentes etapas, según lo expuesto por Quintero-Moreno (2003) y Dorado et al. (2010, 2011, 2013).

Por tanto, en semen fresco, la subpoblación $\mathrm{Sp} 1$ son espermatozoides progresivos que podrían ser espermatozoides que deben ser activados debido a su baja ALH, para luego hiperactivarse en el tracto reproductor de la hembra, o ser células espermáticas que ya perdieron su vigor para movilizarse, aunque tiene trayectorias rectas (Quintero-Moreno et al., 2003; Buzón 2013; Dorado et al., 2013; Acha, 2015; Ortiz, 2016). La $\mathrm{Sp} 2$ son rápidos y progresivos (son los que podrían ser útiles para la fecundación). Podrían ser los espermios con mayor capacidad fecundante, como ha sido propuesto (Quintero-Moreno, 2003; Muiño et al., 2007; Dorado et al., 2010) o estar cerca de la hiperactivación (Dorado et al., 2013). Por las características que muestran las subpoblaciones $\mathrm{Sp} 1$ y Sp2, podrían presentar una mayor resistencia al proceso criogénico (Muiño et al., 2007; Buzón, 2013; Dorado et al., 2013).

La Sp4 son activos, pero no progresivos. Pueden ser células que tienen comprometido su metabolismo y han perdido su progresividad (Quintero-Moreno, 2003; Buzón, 2013; Dorado et al, 2013) o que se encuentran en las últimas etapas de ciclo celular. Así mismo, las Sp3 son células espermáticas lentas y progresivas que pueden tener un compromiso metabólico o están esperando hiperactivación.

En el caso de las subpoblaciones en semen pos-descongelado, la Sp1 corresponde a espermatozoides que tuvieron una mayor resistencia al proceso de criopreservación; 
son muy progresivos y con alto VAP, por lo que podrían ser células aptas para la fertilidad, debido a los altos valores de ALH y BCF (Quintero-Moreno et al., 2003; Buzón 2013; Dorado et al., 2013).

La Sp2 son espermatozoides con movimiento circular o erráticos por sus mínimos valores de LIN y un alto ALH; por lo tanto, son células ya hiperactivadas (Quintero-Moreno, 2003; Dorado et al., 2011). La Sp4 son lentos y tiene una actividad flagelar muy baja. Pueden ser células que tienen comprometido su metabolismo y han perdido su progresividad (Quintero-Moreno et al., 2003; Buzón 2013; Dorado et al., 2013). Las Sp2 y Sp4, por otro lado, son células que ya tienen comprometida, tanto su integridad metabólica como su integridad funcional. Por último, la Sp3 son espermatozoides muy activos, pero con escaso recorrido, aunque también pueden ser células ya hiperactivadas, pero con algún daño a nivel de la cola por su linealidad (altos valores LIN, STR, WOB y BCF), lo que afecta su ALH.

Dorado et al. (2011) definen que la crioinjuria induce una serie de cambios mecánicos, químicos y osmóticos al espermatozoide, causando la muerte de algunas células y comprometiendo aquellas que sobreviven tras el proceso de congelación y descongelación (Watson, 1995, 2000). Los daños causados son lesiones pequeñas que pueden ser acumulativas, resultando en importantes cambios, tanto en la membrana como en el citosol al final del proceso (Nicolas et al., 2012). Por lo tanto, existe una disminución de la viabilidad del espermatozoide que conlleva a una disminución de la capacidad fertilizante (Watson, 2000).

En este estudio, las velocidades espermáticas en las diferentes fases mostraron diferencias significativas entre los procesos $(\mathrm{p}<0.01)$ de VCL, VSL, VAP, LIN, STR, WOB, ALH y BCF. Debido a que la supervivencia, la motilidad y la fecundidad de los espermatozoides disminuyen después del pro- ceso de descongelación, las características del semen fresco se correlacionan pobremente con la calidad del macho (Verstegen et al., 2002). En contraste, una correlación entre ciertas variables de motilidad, anormalidades e integridad del acrosoma pueden ser un mejor predictor de la capacidad fecundante después de la criopreservación (Dorado et al., 2009). Por otro lado, Evans y Maxwell (1990) y Leboeuf et al. (2000) indican que la morfología espermática es un criterio para la selección de los eyaculados fértiles. Eyaculados con un porcentaje bajo de anormalidades morfológicas, antes de la congelación, tienen en promedio, los mejores valores de motilidad y velocidad después de la descongelación (Hidalgo et al., 2007; Vásquez et al., 2013; Rahmatzadeh et al., 2017; Ledesma et al., 2017); sin embargo, MuiñoOtero et al. (2007) concluyeron que los espermatozoides más rápidos y progresivos resisten mejor el efecto dañino de la fase de criopreservación.

Los resultados revelan que los espermatozoides no se distribuyen de forma homogénea en las diferentes subpoblaciones en semen fresco (Sp1: 18.34\%; Sp2: 20.53\%; Sp3: $46.79 \%$ y Sp4: $14.32 \%$ ) y en semen posdescongelación (Sp1: 38.43\%; Sp2: 7.30\%; Sp3: $11.65 \%$ y Sp4: $42.61 \%$ ), lo que coincide con otros hallazgos (Quintero-Moreno, 2003; Ortega-Ferrusola et al., 2009). En todo el proceso, la SP1 cambia de $18.34 \%$ a $38.43 \%$, por lo que se debe considerar lo expuesto por Marín-Briggiler et al., (2002), que algunos procesos, tales como la fosforilación de la tirosina de las proteínas del esperma, son preponderantes frente a la viabilidad del esperma y en la adquisición de movilidad progresiva rápida. Una dependencia de la temperatura para el aumento de la hiperactividad espermática está asociada con aumento en la movilidad progresiva rápida y capacitación espermáticas (Muiño-Otero, 2008).

Para el parámetro de linealidad (LIN), Cox et al. (2006) determinaron que existe una alta correlación con los demás parámetros 
de motilidad evaluados por el sistema CASA para semen caprino, demostrando que valores mayores a $50 \%$ en espermatozoides evaluados in vitro presentan una excelente migración. Adicionalmente, parámetros como LIN y ALH parecen ser indicadores de la hiperactivación de los espermatozoides (Peña y Linde-Forsberg, 2000). Sin embargo, para Quintero-Moreno et al. (2003), un valor bajo (menor a 50\%) está correlacionado con la hiperactivación espermática. Hernández-Corredor et al. (2013) encontraron valores de LIN de $48 \%$ y STR de $72.8 \%$. Anand y Yadav (2016) encontraron valores de LIN (29.6\%) y STR $(61.62 \%)$ parecidos en semen descongelado, lo que sugiere que un LIN menor a $30 \%$ en caprinos es indicativo de hiperactividad y STR menores de $50 \%$ puede explicarse por los tamaños de la cabeza de los espermatozoides (espermatozoides de cabezas largas dan un valor mayor a células espermáticas con cabezas anchas).

\section{Conclusiones}

Con el uso del sistema CASA y el análisis multivariante se logró identificar cuatro subpoblaciones espermáticas con características diferentes de movimiento, definidas en el eyaculado fresco de los caprinos. Al someter estas células al proceso de criopreservación se modificó significativamente la distribución de espermatozoides dentro de las subpoblaciones, evidenciándose la crioinjuria sufrida y como se adaptan las células espermáticas a esta temperatura extrema.

\section{Literatura Citada}

1. Abaigar T, Holt WV, Harrison RA, del Barrio G 1999. Sperm subpopulations in boar (Sus scrofa) and gazelle (Gazella dama mhorr) semen as revealed by pattern analysis of computerassisted motility assessment. Biol Reprod 60:32-41. doi: 10.1095/biolreprod60.1.32
2. Acha D. 2015. Optimización de la metodología de preservación del esperma de asno andaluz (Equus asinus). Tesis doctoral. Córdoba, España: Univ. de Córdoba. 99 p.

3. Anand M, Yadav S. 2016. Assessment of motion and kinematic characteristics of frozen-thawed Sirohi goat semen using computer-assisted semen analysis. Vet World 9: 203-206. doi: 10.14202/ vetworld.2015.203-206

4. Boyers SP, Davis RO, Kats DF. 1989. Automated semen analysis. Curr Prob Obst Gyn F 12: 172-200.

5. Buzón A. 2013. Análisis cinético del espermatozoide del caballo bajo el sistema Sperm Class Analyzer. Tesis doctoral. Córdoba, España: Univ. de Córdoba. 172 p.

6. Chandler JE, Painter CL, Adkison RW, Memon MA, Hoyt PG. 1988. Semen quality characteristics of dairy goats. J Dairy Sci 71: 1638-1646. doi: 10.3168/ jds.S0022-0302(88)79728-3

7. Cox JF, Alfaro V, Montenegro V, Rodríguez-Martínez H. 2006. Computer-assisted analysis of sperm motion in goats and its relationship with sperm migration in cervical mucus. Theriogenology 66: 860-867. doi: 10.1016/j.theriogenology.2006.01.062

8. Davis RO, Katz DF. 1993. Operational standards for CASA instruments. J Androl 14: 385-394.

9. Dorado J. 2003. Respuesta a la congelación-descongelación del esperma de macho cabrío. Tesis Doctoral. España: Univ. de Córdoba. 203 p.

10. Dorado J, Rodríguez I, Hidalgo M. 2007. Cryopreservation of goat spermatozoa: comparison of two freezing extenders based on post-thaw sperm quality and fertility rates after artificial insemination. Theriogenology 68: 168177. doi: 10.1016/j.theriogeno-logy.2007.04.048

11. Dorado J, Hidalgo M, Muñoz A, Rodríguez I. 2009. Assessment of goat semen freezability according to the spermatozoa characteristics from fresh and frozen samples. Anim Reprod Sci 
112: 150-157. doi: 10.1016/j.anireprosci.2008.04.005

12. Dorado J, Muñoz-Serrano A, Hidalgo $M$. 2010. The effect of cryopreservation on goat semen characteristics related to sperm freezability. Anim Reprod Sci 121: 115-123. doi: 10.1016/ j.anireprosci.2010.04.182

13. Dorado J, Gálvez M, Murabito M, Muñoz-Serrano A, Hidalgo M. 2011. Identification of sperm subpopulations in canine ejaculates: effects of cold storage and egg yolk concentration. Anim Reprod Sci 127: 106-113. doi: 10.1016/ j.anireprosci.2011.07.005

14. Dorado J, Acha D, Ortiz I, Gálvez MJ, Carrasco JJ, Díaz B, Gómez-Arrones $V$, et al. 2013. Relationship between conventional semen characteristics, sperm motility patterns and fertility of Andalusian donkeys (Equus asinus). Anim Reprod Sci 143: 64-71. doi: 10.1016/j.anireprosci.2013.10.003

15. Dorado J, Acha D, Gálvez MJ, Ortiz I, Carrasco JJ, Díaz B, GómezArrones V, et al. 2013. Sperm motility patterns in Andalusian donkey (Equus asinus) semen: effects of body weight, age, and semen quality. Theriogenology 79: 1100-1109. doi: 10.1016/ j.theriogenology.2013.02.006

16. Esteso MC, Rodríguez E, Toledano Díaz A, Castaño C, Pradiee J, LópezSebastián A, et al. 2015. Descriptive analysis of sperm head morphometry in Iberian ibex (Capra pyrenaica): optimum sampling procedure and staining methods using Sperm Class Analyzer®. Anim Reprod Sci 155: 42-49. doi: 10.1016/j.anireprosci.2015.01.014

17. Evans G, Maxwell W. 1990. Salamon's artificial insemination of sheep and goats. London: Butterworth. $194 \mathrm{p}$.

18. Hernández-Corredor L, Nivia-Osuna A, Hernández-Villamizar D, RubioParada J, Quintero-Moreno A. 2013. Evaluación de la movilidad espermática de semen caprino criopreservado bajo diferentes medios diluyentes a través del sistema CASA. Respuesta 2: 16-27.
19. Hernández-Corredor L, Dorado J, Quintero-Moreno A, Ortiz I, Buzón A, Corzo M, et al. 2014. Efecto de dos diluyentes a base de lecitina de soya sobre parámetros morfométricos en semen caprino. Rev Sennova 1: 30-43

20. Hidalgo M. 2004. Estudio del efecto de la congela-ción-descongelación sobre los parámetros morfomé-tricos del espermatozoide de macho cabrío. Tesis Doctoral. España: Univ. de Córdoba. 225 p.

21. Hidalgo M, Rodríguez I, Dorado J. 2006. Influence of staining and sampling procedures on goat sperm morphometry using the Sperm Class Analyzer. Theriogenology 66: 996-1003. doi: 10.1016/j.theriogenology.2006.02.039

22. Hidalgo M, Rodríguez I, Dorado J. 2007. The effect of cryopreservation on sperm head morphometry in Florida male goat related to sperm freezability. Anim Reprod Sci 100: 61-72. doi: 10.1016/ j.anireprosci.2006.07.003

23. Hoflack G, Opsomer G, Rijsselaere T, Van Soom A, Maes D, de Kruif A, Duchateau L. 2007. Comparison of computerassisted sperm motility analysis parameters in semen from Belgian blue and Holstein-Friesian bulls. Reprod Domest Anim 42: 153-161. doi: 10.1111/ j.1439-0531.2006.00745.x

24. Holt WV. 1996. Can we predict fertilty rates? Making sense of sperm motility. Reprod Domest Anim 31: 17-24. doi: 10.1111/j.1439-0531.1995.tb00001.x

25. Holt WV, Harrison RA. 2002. Bicarbonate stimulation of boar sperm motility via a protein kinase A-dependent pathway: between-cell and betweenejaculate differences are not due to deficiencies in protein kinase A activation. J Androl 23: 557-565. doi: 10.1002/j.1939-4640.2002.tb02279.x

26. Kamal A, Gubartallah A, Ahmed A, Bakhiet O, Babiker A. 2005. Comparative studies on reproductive performance of Nubian and Saanen bucks under the climatic conditions of Khartoum. J Anim Vet Adv 4: 942-944. 
27. Karagiannidis A, Varsakeli S, Karatzas $G$. 2000. Characteristics and seasonal variations in the semen of Alpine, Saanen and Damascus goat bucks born and raised in Greece. Theriogenology 53: 1285-1293. doi: 10.1016/S0093-691X(00)00272-7

28. Leboeuf B, Restall B, Salamon S. 2000. Production and storage of goat semen for artificial insemination. Anim Reprod Sci 62: 113-141. doi: 10.1016/ S0378-4320(00)00156-1

29. Ledesma A, Zalazar L, FernándezAlegre E, Hozbor F, Cesari A, Martínez-Pastor F. 2017. Seminal plasma proteins modify the distribution of sperm subpopulations in cryopreserved semen of rams with lesser fertility. Anim Reprod Sci 184: 44-50. doi: 10.1016/ j.anireprosci.2017.06.015

30. Marín-Briggiler C, Tezon J, Miranda P, Vázquez L, Levin M. 2002. Effect of incubating human sperm at room temperature on capacitation-related events. Fertil Steril 77: 252-259. doi: 10.1016/S0015-0282(01)02982-X

31. Martínez-Pastor F, Johnnisson A, Gil J, Kaabi M, Anel L, Paz P, RodríguezMartínez H. 2004. Use of chromatin stability assay, mitochondrial stain JC-1, and fluorometric assessment of plasma membrane to evaluate frozen-thawed ram semen. Anim Reprod Sci 84: 121133. doi: 10.1016/j.anireprosci.2003.12.006

32. Martinez-Pastor F, Garcia-Macias V, Alvarez M, Herraez, P, Anel L, de Paz P. 2005. Sperm subpopulations in Iberian red deer epididymal sperm and their changes through the cryopreservation process. Biol Reprod 72: 316-327. doi: 10.1095/biolreprod.104.032730

33. Martínez-Pastor F, Tizado EJ, Garde JJ, Anel L, de Paz P. 2011. Statistical series: opportunities and challenges of sperm motility subpopulation analysis. Theriogenology 75: 783-795. doi: 10.1016/j.theriogenology.2010.11.034
34. Mortimer ST. 1997. A critical review of the physiological importance and analysis of sperm movement in mammals. Hum Reprod Update 3: 40339. doi: 10.1093/humupd/3.5.403

35. Mortimer ST. 2000. CASA - Practical aspects. J Androl 21: 515-524. doi: 10.1002/j.1939-4640.2000.tb02116.x

36. Muiño-Otero R. 2008. Evaluación de la motilidad y viabilidad del semen bovino mediante el uso de sistemas CASA y citometria de flujo: identificación de subpoblaciones espermáticas. Tesis Doctoral. España: Univ. de Santiago de Compostela. $103 \mathrm{p}$.

37. Muiño-Otero R, Fernández M, Peña $A .2007$. Post-thaw survival and longevity of bull spermatozoa frozen with an egg yolk-based or two egg yolk-free extenders after an equilibration period of 18h. Reprod Domest Anim 42: 305-311. doi: 10.1111/j.1439-0531.2006.00784.x

38. Nicolas M, Alvarez M, Borragan S, Martínez-Pastor F, Chamorro C, Alvarez-Rodríguez M, et al. 2012. Evaluation of the qualitative and quantitative effectiveness of three media of centrifugation (Maxifreeze, Cushion Fluid Equine, and PureSperm 100) in preparation of fresh or frozenthawed brown bear spermatozoa. Theriogenology 77: 1119-1128. doi: 10.1016/j.theriogenology.2011.10.016

39. Ortega-Ferrusola C, GonzálezFernández L, Macías-García B, Salazar-Sandoval $C$, MorilloRodríguez A, Rodríguez-Martínez, $\mathrm{H}$, et al. 2009. Effect of cryopreservation on nitric oxide production by stallion spermatozoa. Biol Reprod 81: 1106-1111 doi: 10.1095/biolreprod.109.078220

40. Ortiz I. 2016. Efecto de la selección de espermatozoides mediante centrifugación coloidal sobre la calidad de las dosis de semen de asno refrigeradas y congeladas. Tesis Doctoral. Córdoba, España: Univ. de Córdoba. 167 p.

41. Peña A, Linde-Forsberg C. 2000. Effects of Equex, one or two step dilution- 
and two freezing thawing rates on postthaw survival of dog spermatozoa. Theriogenology 5: 859-875. doi: 10.1016/ S0093-691X(00)00397-6

42. Quintero-Moreno A. 2003. Estudio sobre la dinámica de poblaciones espermáticas en semen de caballo, cerdo y conejo. España: Univ. Autónoma de Barcelona. $164 \mathrm{p}$.

43. Quintero-Moreno A, Miró J, Rigau T, Rodríguez-Gil JE. 2003. Identification of sperm subpopulations with specific motility characteristics in stallion ejaculates. Theriogenology 59: 19731990. doi: 10.1016/j.anireprosci.2011.10.005

44. Rahmatzadeh M, Kohram H, ZareShahneh A, Seifi-Jamadi A, Ahmad E. 2017. Antioxidative effect of BHA in soya bean lecithin-based extender containing glycerol or DMSO on freezing capacity of goat semen. Reprod Domest Anim 28: 1-7. doi: 10.1111/rda. 13007

45. Ramón M, Soler AJ, Ortiz JA, García Álvarez O, Maroto Morales A, Roldan ERS, Garde JJ. 2013. Sperm population structure and male fertility: an intraspecific study of sperm design and velocity in red deer. Biol Reprod 89: 1-7. doi: 10.1095/biolreprod.113.112110

46. Salomón A. 2011. Capacitación del espermatozoide de caprino durante el proceso de estabilización pre criopreservación. Tesis de Médico Veterinario. Veracruz, México: Univ. Veracruzana. 50 p.
47. Thurston LM, Watson PF, Mileham AJ, Holt WR. 2001. Morphologically distinct sperm subpopulations defined by Fourier shape descriptors in fresh ejaculates correlate with variation in boar semen quality following cryopreservation. J Androl 22: 382-94. doi: 10.1002/j.19394640.2001.tb02194.x

48. Vásquez J, Núñez V, Florentini E, Gonzales J, Camargo L, y Valdivia M. 2013. Effects of five cryoprotective agents on quality of sheep epididymal spermatozoa during pre-freezing. Livestock Sci 152: 94-99. doi: 10.1016/ j.livsci.2012.12.011

49. Verstegen J, Iguer-Ouada M, Onclin K. 2002. Computer assisted semen analyzer in andrology research and veterinary practice. Theriogenology 57 : 149-179. doi: 10.1016/S0093-691X(01)00664-1

50. Watson P. 1995. Recent developments and concepts in the cryopreservation of spermatozoa and the assessment of their post-thawing function. Reprod Fertil Dev 7: 871-891.

51. Watson P. 2000. The causes of reduced fertility with cryopreserved semen. Anim Reprod Sci 60-61: 481-492. doi: 10.1016/ S0378-4320(00)00099-3

52. Zamfirescu S, Nadolu D, Andreea A. 2005. The influence of the glycerol levelupon the freezing pretability of Saanen and Alpine bucks semen. Seria Zootehnie 52: 1-5. 